\title{
Continuous apparent motion by successive presentation of sound for miscellaneous sources
}

Ikuichiro Kinoshita $^{\dagger}$ and Shigeaki Aoki

NTT Human Interface Laboratories,

1-1, Hikari-no-oka, Yokosuka, 239 Japan

(Received 7 December 1996)

Keywords : Hearing, Apparent motion, Burst duration, Target setting PACS number: 43. 55. Hy, 43. 66. Lj, 43. 66. Qp

\section{Introduction}

The perception of motion has become a subject of interest in the production of a virtual sound environment. Even for simulating a moving sound the use of stationary sound drivers may be feasible. One solution is successive presentation. It may be the simplest method to produce apparent motion. However, the most significant condition is the timing of presentation rather than the distribution (e.g. Nakajima et al., 1990 ; Strybel and Neale, 1994). Continuous motion is usually observed for burst durations longer than $20 \mathrm{~ms}$. It is maintained, as long as the burst duration of longer than $50 \mathrm{~ms}$ increases more quickly than the increase of interstimulus onset intervals (ISOIs). In other words, a silence gap or a longer burst duration (even equal to the ISOI) collapses the impression of continuous motion (Mizushima et al., 1994).

Note that the above-mentioned performance was obtained using an unusual source for listeners (such as white noise), although it specifies a criterion for target setting in a 3D acoustical environment. The target setting uses discrete positions to give clues as to the sound position for the listener to localize it as a function of time. Namely, motion is transcribed as successive updates of the position. For implementation of continuous motion, investigation of usual sounds (such as voice) assists in estimating the timing specification.

The present paper investigates particularly the upper thresholds of burst duration for continuous apparent motion using unusual and usual sources as stimuli. The lower threshold of $20 \mathrm{~ms}$ is insufficient to represent temporal characteristics that produce sensations such as fluctuation or give clues to phonetic or semantic information (Zwicker and Fastl, 1990).

\section{Method}

\subsection{Subjects}

Four people aged 25-36 with normal hearing participated as subjects. Two of the subjects were the authors. The other two had no previous knowledge of this experiment. Here, they are referred to as subjects

\footnotetext{
${ }^{\dagger}$ Present address: 3-9-11, Midori-cho, Musashino, 180 Japan
}

$\mathrm{a}, \mathrm{h}, \mathrm{k}$ and $\mathrm{s}$.

2.2 Setup

Figure 1 illustrates the experimental setup and the disposition of loudspeakers and subject. The setup was composed of a workstation, a digital-to-analog (D/ A) converter, low-pass and high-pass filters and a power amplifier. The workstation was used to store digital sequences in data files and to control the testing procedure. Each of the digital sequences was a source for stimuli. It was forwarded from the data file to the D/A converter and transformed into analog signals at a sampling rate of $16 \mathrm{kHz}$. The output analog signal from the D/A converter was amplified and delivered to the loudspeakers through each channel, after filtering the components of frequencies from 0.2 to $7.0 \mathrm{kHz}$. Tests were conducted in a large isolated anechoic room. An array of loudspeakers at a spacing of 30 degrees was arranged in the $1.5 \mathrm{~m}$ radius of a circle centering on the subject. However, the three positioned in front of the subject of the twelve loudspeakers, - the center one and the two adjacent to the center-were actuated as shown in Fig. 2.

\subsection{Stimuli}

Stimuli were successively presented from the loudspeaker at one end to the one at the other end, either clockwise or counter-clockwise as depicted in Fig. 2. The burst duration for each stimulus $T$ was variable for each trial, including $10 \mathrm{~ms}$ of either sinusoidal rise at the beginning or decay at the end. The average level of each stimulus was adjusted to be $65 \mathrm{~dB}$ (A-weighted) at the subject location under a free field.

The examined sources were as follows: (a) white noise, (b) pink noise, (c) one-third octave band noise with middle frequency $1.0 \mathrm{kHz}$, (d) female voice, (e) stationary noise with a speech-like spectrum, (f) broadband noise with a speech-like temporal envelope, (g) voice with silent gaps removed and (h) temporally reversed voice. Source (a) was a pseudo-random sequence generated on the work station. Sources (b) and (c) were recorded from the compact disk "AUDIO TEST CD-1" produced by the Japan Audio Society (JAS). Source (d) was a portion of female speech from another disk, named "IMPACT 2" also from the JAS. Source (e) was synthesized by convolving source (a) 


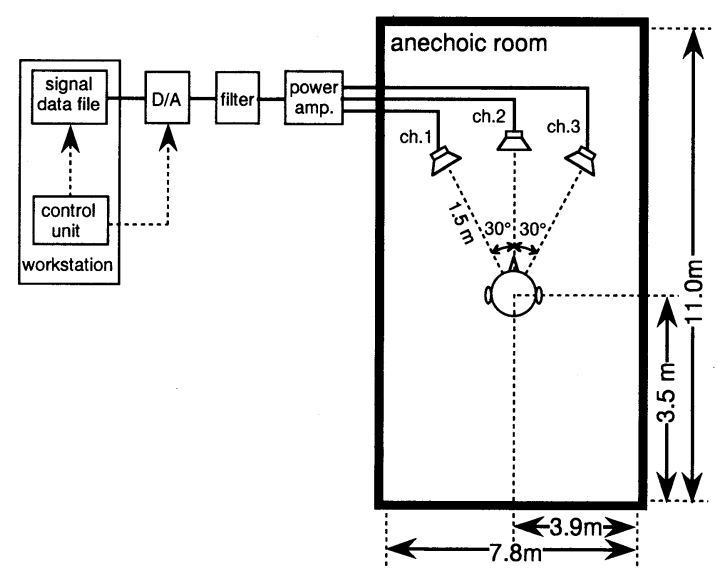

Fig. 1 Illustration of setup composed of a workstation, D/A converter, filter and power amplifier, and location of actuated loudspeakers and the subject in an anechoic room.

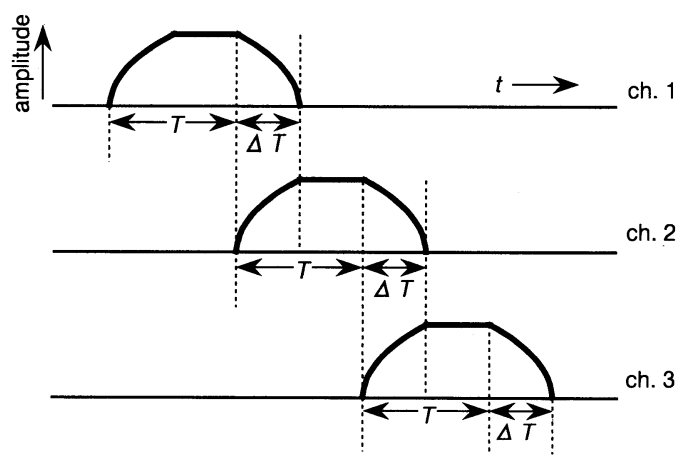

Fig. 2 Envelopes for the stimuli tested, presented in succession from ch. 1 to ch. 3 at burst duration $T$ with both rise and decay duration $\Delta T$ of $10 \mathrm{~ms}$.

with a filtering coefficient characterizing the power spectrum of source (d). The coefficient was transformed from the power spectrum averaged over 64 sets of 1,024 samples of source (d). Source (f) was the product of source (a) and source (d). Source (g) was a rearrangement of source (d), with the silent gaps curtailed. Source $(\mathrm{h})$ was a reversed sequence of source (d).

\subsection{Procedures}

The subjects were individually tested during a single trial block, in which one source was examined. The order of sources was randomized and differentiated for each subject. In each trial block, 110 trials were conducted ; 10 trials for each of 11 burst durations. Burst duration for each trial was randomly selected from the 11 burst durations, which were set in linearly equal intervals between the maximum and the minimum. First we determined the maximum and the minimum where all the subjects obviously perceived the sound travelling successively and continuously, respectively. For example, the maximum and the minimum for white noise were $100 \mathrm{~ms}$ and $50 \mathrm{~ms}$. The sequence of stimulus presentation, whether clockwise or counterclockwise, was also shuffled at random for each trial. Five trials of common burst duration and presentation sequence were conducted in a trial block. After each trial, the subject was instructed to judge whether the sound appeared to be moving continuously or not.

\section{Results and discussions}

\subsection{Psychometric function}

The percentage of responses for continuous motion, collected from subject $\mathrm{k}$. for source (b), is shown as a function of burst duration in Fig. 3. This example illustrates a typical distribution of responses where sounds presented with a short burst duration close to the minimum tended to be perceived moving continuously. In contrast, sounds presented with a long burst duration near the maximum were perceived to be moving brokenly or in succession. Thus, the upper threshold of burst duration for continuous apparent motion was defined by using a $50 \%$ criterion.

3.2 Upper threshold of burst duration

The upper threshold of burst duration for continuous apparent motion is plotted for each source and each subject in Fig. 4. It ranges from $72 \mathrm{~ms}$ for source (a) to $580 \mathrm{~ms}$ for source (d). An analysis of variance on these data indicates a significant effect of source $(F(7,21)=16.71 ; p<0.001)$, but no significant effect of subject $(F(3,21)=2.487 ; p>0.05)$.

The present result shows that the upper threshold for sources (a) and (b), which carry a broad band of frequency components, is shortest. The short upper threshold of $72 \mathrm{~ms}$ is consistent with interpolation of

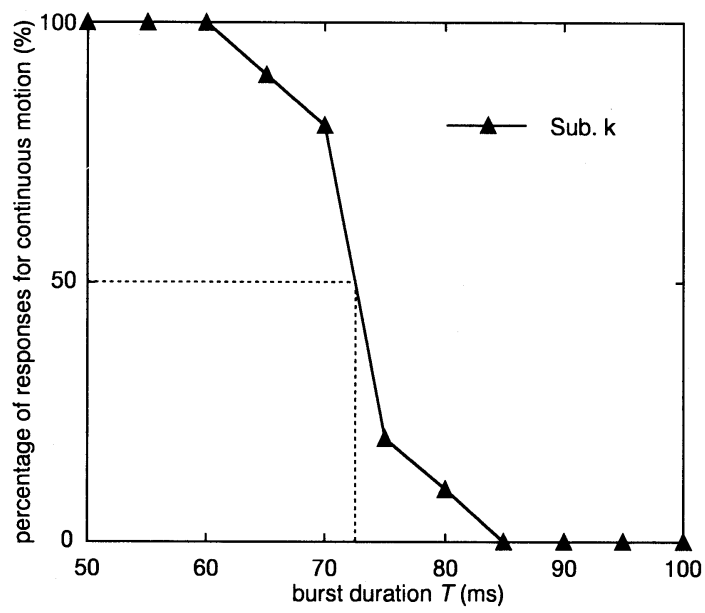

Fig. 3 An example of psychometric function of burst duration $T$ based on responses from subject $\mathrm{k}$ for source (b). The upper threshold was defined at $50 \%$ for continuous motion. 


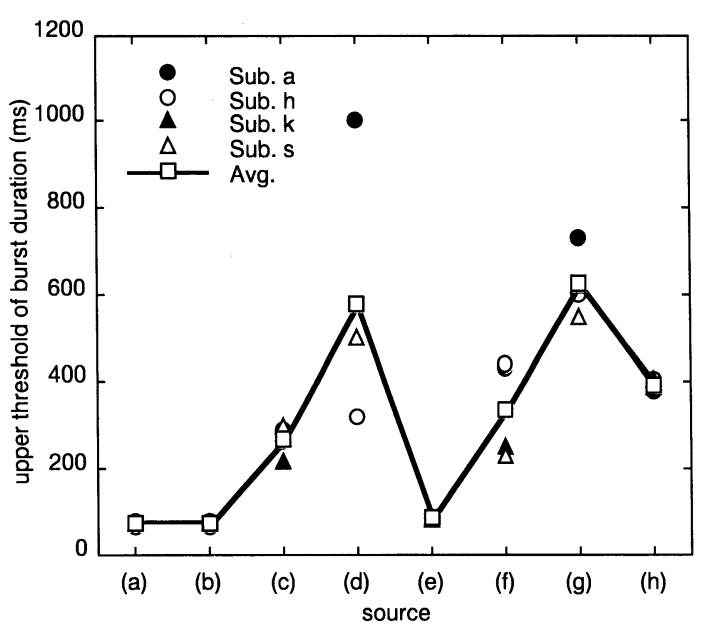

Fig. 4 Upper threshold of burst duration for continuous motion (in $\mathrm{ms}$ ) plotted as a function of source for each subject. The solid line links the averages. Tested sources are as follows, (a) white noise, (b) pink noise, (c) onethird octave band noise with middle frequency $1.0 \mathrm{kHz}$, (d) female voice, (e) stationary noise with speech-like spectrum, (f) broadband noise with speech-like temporal envelope, $(\mathrm{g})$ voice with silent gaps removed and (h) temporally reversed voice.

previous research on various ISOI thresholds, which fix the boundaries between continuous, broken and successive perceptions for burst duration. This signifies that the short threshold confines the range of possible burst durations for continuous apparent motion. For implementation of continuous motion, it is recommended that the target position be renewed at intervals of less than $72 \mathrm{~ms}$.

As for the difference of the upper threshold among sources, they are discussed in the following groups: Group I covers sources (a), (b) and (e), Group II covers sources (c), (f) and (h) and Group III covers sources (d) and $(\mathrm{g})$. In each group, no significant differences are observed $(t(3)>2.64 ; p>0.05)$. The upper thresholds for group I were short and showed little individual variation compared with those for other groups. With regards to the considerable difference among sources (c), (d) and (e), the frequency band alone does not explain the long upper thresholds of other groups of sources. One might attribute the factor to the perception of fluctuation. Sources of group I have all a stationary temporal envelope, while the others have a modulated one, which produces the fluctuation. This is relevant to the perception for modulated sound
(Zwicker and Fastl, 1990); the strength of fluctuation remains at low-modulation frequencies up to around 20 $\mathrm{Hz}$.

The upper thresholds for group II are still shorter than those for group III. The difference of characteristics between the both groups is whether or not phonetic and semantic informations is carried. Not only source (d), but also source (g) was recognized as voice. With respect to the insignificant difference between sources (d) and (g), silent gaps in voice, unlike those in broadband noises, do not encourage broken motion nor successive perception. This implies that the perception for amplitude modulation alone is insufficient to explain the long upper thresholds for group III.

Nevertheless the above justification would be premature without evidence focused on the respective factors. Confirmation of the results here requires systematical investigation into one of the physical characteristics of the source, such as amptitude modulation.

\section{Summary}

The upper threshold of burst duration for continuous apparent motion was measured. Stimuli were successively presented using three loudspeakers arranged in front of the subject with spacings of 30 degrees. The following sources were employed for the stimuli: (a) white noise, (b) pink noise, (c) one-third octave band noise with middle frequency of $1.0 \mathrm{kHz}$, (d) female voice, (e) stationary noise with a speech-like spectrum, (f) broadband noise with a speech-like temporal envelope, (g) voice with silent gap removed and (h) temporally reversed voice.

As a result, the upper threshold significantly differs among sources and ranges from $72 \mathrm{~ms}$ to $580 \mathrm{~ms}$. Sources (a) and (b) produce the shortest upper threshold, that specifies a update condition of moving target setting.

\section{References}

Mizushima, K., Nakanishi, S., and Morimoto, M. (1994). "Continuity of a moving sound image caused by successive signals from two discretely located loudspeakers," J. Acoust. Soc. Jpn. (E) 15, 179-188.

Nakajima, T., Tamaribuchi, K., and Saito, S. (1990). "Perception of the motional image induced from distributed sound sources excited sequentially," Tech. Rep. Hear. Acoust. Soc. Jpn. H-90-6, 1-6 (in Japanese).

Strybel, T.Z. and Neale, W. N. (1994). "The effect of burst duration, interstimulus onset interval, and loudspeaker arrangement on auditory apparent motion in the free field," J. Acoust. Soc. Am. 96, 3463-3475.

Zwicker, E. and Fastl, H. (1990). "Fluctuation strength," in Psychoacoustics, E. Zwicker and H. Fastl, Eds. (Springer-Verlag, Berlin, 1990), Chaps. 10 and 11, pp. $222-236$ 